\title{
MONNAIE ET RICHESSE CHEZ JOHN LOCKE UNE POLITIQUE DE L'ÉCONOMIE
}

\author{
Isabelle GARO
}

RÉSumÉ : Les travaux de Locke sur la monnaie ne sont pas une esquisse maladroite des théories économiques libérales ultérieures mais une partie intégrante de sa philosophie. Il s'agit alors de montrer que l'étude lockienne de la monnaie se situe à l'intersection de trois axes de recherche distincts. D'une part, s'inspirant de l'analyse mercantiliste de la richesse et de sa circulation, Locke s'efforce de définir les catégories propres de l'analyse monétaire. D'autre part, la monnaie métallique se définissant à la fois comme marchandise et comme signe, le problème se situe alors sur le terrain d'une analyse philosophique de la représentation, qui met en jeu la distinction entre substance, mode mixte et relation établie dans l'Essai philosophique concernant l'entendement humain. Enfin, la monnaie assure la liaison entre l'activité privée et l'ensemble de la vie sociale : Locke examine son rôle du point de vue de la propriété, du travail et des finalités collectives qu'ils doivent réaliser. Au total, la monnaie apparait bien comme un problème crucial de la pensée lockienne, et non comme un thème annexe et conjoncturel.

Mots-CLÉs : monnaie, échange, représentation, travail, propriété.

ABSTRACT: Locke's works on money are not the clumsy outline of the liberal economic theories that developed later on but they are part and parcel of his philosophy. This study will then show that Locke's works on money are at the crossroads of three distinct lines of research. Firstly, inspired by the mercantilist analysis of wealth and its circulation, Locke endeavours to define the categories proper to monetary analysis. Secondly, metallic money being defined both as commodity and symbol, the problem then stands on the ground of a philosophical analysis of the notion of representation which brings into play the distinction, established in the Essay concerning human understanding, between substance, mixed mode and relation. Lastly, money provides the link between private activity and the whole of social life; so Locke examines its role from the point of view of property, labour and the collective aims both must fulfil. On the whole, money does appear to be a crucial issue in the thought of Locke, not a subsidiary theme due to circumstances.

KEYWORDS : money, exchange, representation, labour, property.

Revue de synthèse : $4^{\mathrm{e}}$ S. $\mathrm{n}^{\text {os }} 1-2$, janv.-juin 2000, p. 9-43. 
Zusammenfassung: Lockes Arbeiten über das Geld sind nicht etwa eine ungeschickte Skizze der späteren liberalen Wirtschaftstheorien, sondern ein substantieller Bestandteil seiner Philosophie. Es gilt daher zu zeigen, dass die Locke'sche Untersuchung über das Geld im Schnittpunkt dreier distinkter Forschungsrichtungen steht. Einerseits bemüht sich Locke, die eigentlichen Kategorien der Geldanalyse zu definieren, wobei er sich bei der merkantilistischen Analyse des Reichtums und seines Flusses inspiriert. Da sich das Geld als Metall gleichzeitich als Ware und als Zeichen definieren lässt, liegt das Problem aber anderseits im Bereich der philosophischen Analyse der Vorstellung, die sich auf die Unterscheidung zwischen Substanz, gemischtem Modus und Verhältnis bezieht, wie sie in der Essay über den menschlichen Verstand dargelegt ist. Schliesslich ist das Geld Träger der Verbindung zwischen privater Handdlung und der Gesamtheit des gesellschaftlichen Lebens : Locke untersucht seine Rolle unter dem Gesichtspunkt des Eigentums, der Arbeit und der mit ihm angestrebten kollektiven Ziele. Zusammenfassend stellt im Locke'schen Verständnis das Geld nicht etwa ein nebensächliches und konjunkturelles Thema, sondern ein zentrales Problem dar.

STICHWÖRTER: Geld, Austausch, Vorstellung, Arbeit, Eigentum.

RESUMEN : Los trabajos de Locke sobre la moneda no son un torpe esbozo de las posteriores teorías económicas liberales, sino parte integrante de su filosofía. Se trata de mostrar, en consecuencia, que el análisis lockiano de la moneda se sitúa en la intersección de tres líneas de investigación distintas. Por una parte, Locke intenta definir las categorías propias del análisis monetario inspirándose en el análisis mercantilista de la riqueza y su circulación. Por otra, la cuestión se plantea en los términos propios de un análisis filosófico de la representación al definirse la moneda metálica a la vez como mercancía y signo, lo que pone en juego la distinción entre sustancia, modo mixto y relación establecida en el Ensayo filosófico sobre el entendimiento humano. Finalmente, la moneda asegura la vinculación entre la actividad privada y el conjunto de la vida social : Locke examina su papel desde el punto de vista de la propiedad, del trabajo, y de los fines colectivos que éstos deben realizar. En suma, la moneda se presenta como un problema central del pensamiento lockiano, y no como un tema secundario y circunstancial.

PALABRAS CLAVES : moneda, intercambio, representación, trabajo, propiedad.

Isabelle Garo, née en 1963, est agrégée de philosophie et docteur de $3^{\mathrm{e}}$ cycle. Elle enseigne au lycée Pierre-Bayen à Châlons-en-Champagne et ses recherches portent sur Marx et l'économie politique classique.

Adresse : Centre d'histoire des systèmes de pensée modernes, UFR de philosophie de l'université Paris-I, 17 rue de la Sorbonne, 75231 Paris Cedex 05.

Courrier électronique : igaro@club-internet.fr 
Les travaux de John Locke sur la monnaie sont l'objet d'un curieux paradoxe : beaucoup des commentateurs qui ont vu dans son œuvre la première expression du libéralisme économique se sont surtout intéressés au chapitre du Second traité concernant la propriété et ont délaissé, voire ignoré, les essais consacrés à la monnaie et au taux d'intérêt ${ }^{1}$. De leur côté, la plupart des historiens de la pensée économique ${ }^{2}$ présentent Locke comme un théoricien important, souvent comme l'un des promoteurs de la théorie quantitative, mais signalent que ses confusions et ses incohérences l'ont empêché de parvenir à une analyse consistante de la réalité économique dans son ensemble. Locke serait resté à mi-chemin sur la voie d'une analyse économique en cours d'émancipation à l'égard des tenants et des aboutissants politiques et moraux qui caractérisaient la pensée économique mercantiliste.

Or la lecture des essais sur la monnaie révèle l'insuffisance de cette interprétation et met en lumière toute la complexité du rapport de Locke ${ }^{3}$ au libéralisme politique et économique ultérieur: loin d'être l'esquisse d'une théorie économique, malheureusement encore grevée par l'hypothèque de considérations politiques et métaphysiques, la pensée lockienne construit là un pan important d'une philosophie d'ensemble, non systématique certes, mais dont il paraît plus fructueux de présumer la cohérence que de dénoncer rétrospectivement la faiblesse au regard des instruments d'analyse économique ultérieurement élaborés. L'étude des pamphlets économiques de Locke met bien plutôt en lumière la façon dont les catégories économiques ne s'élaborent que dans la proximité la plus extrême avec une philosophie morale et politique et une théorie de la connaissance. À cet égard, Locke est, du fait même de son statut de philosophe, un exemple remarquable de la façon dont l'étude de la monnaie se nourrit de toutes les autres élaborations théoriques et y fait retour par ses apports spécifiques, et cela dans le cadre d'une pensée générale de la représentation, de ses origines et de sa fonction. La monnaie est moins un objet spécial voué à l'étude technique de ses propriétés, qu'un échangeur théorique, qui ouvre l'analyse économique aux dimensions politiques, théologiques, éthiques et philosophiques des choix qui s'opèrent sur son terrain.

1. Notamment: Strauss, 1986; Macpherson, 1973; Dumont, 1977.

2. P. ex. : Blaug, 1987, p. 20-26; Guggenheim, 1978, p. 35 ; Hyde Kelly, 1991, p. $105-$ 106; Pribram, 1986, p. 70; SChumpeter, 1983, p. 407.

3. Pour un état des lieux des études lockiennes et concernant en particulier la question du libéralisme, on consultera : Spitz, 1994a et 1994b. Cf. également Pocock, 1980. 
Les essais économiques de Locke doivent bien entendu être replacés dans le contexte historique et politique de leur rédaction, qui se situe entre 1668 et 1695. En 1668, Locke est déjà au service de Shaftesbury, alors chancelier de l'Échiquier et qui se consacre tout spécialement à l'administration et au commerce colonial. En 1695, ses conseils ont conduit le parlement whig à la décision de refonte de la monnaie d'argent et Locke a pris part à la fondation de la Banque d'Angleterre. Entre ces deux dates, l'Angleterre connaît un manque de liquidités qui provoque une crise de circulation et une forte hausse des prix, ainsi qu'un vif débat national portant sur le remboursement de la dette publique, le prélèvement des impôts, le soutien de l'État au commerce anglais, dans un contexte international de guerre et de rivalité avec les autres pays européens, et notamment la Hollande. S'il est permis de voir là la cause occasionnelle des premières œuvres économiques de Locke, il est clair aussi que leur objet ne se réduit pas à la défense et illustration de thèses partisanes dictées à la fois par la conjoncture politique et l'appartenance à un camp d'intérêt.

Â cet égard, la position de Locke est d'ailleurs originale : Richard Aschcraft a montré que la stratégie de l'aile radicale de l'opposition anglaise durant la Restauration vise à sceller une alliance entre les artisans, les boutiquiers et les propriétaires terriens ${ }^{4}$. Or Locke, outre la place importante qu'il accorde au peuple, adresse de vives critiques aux marchands, aux banquiers et même à la gentry, dont il défend pourtant le plus souvent les intérêts. Et s'il insiste sur les bénéfices collectifs qu'on peut attendre du développement du commerce, celui-ci n'est pas à lui seul la panacée en matière sociale: bien des passages des pamphlets économiques soulignent les divergences fondamentales d'intérêt entre catégories sociales, divergences que les décisions de politique économique doivent précisément prendre en considération ${ }^{5}$.

Les analyses économiques de Locke ne peuvent donc être réduites à un ensemble d'affirmations tactiques. Elles présentent une véritable ambition théorique, qui n'annule pas mais complète et élargit leur dimension conjoncturelle. La précision de la discussion lockienne sur les questions

4. AshCRAFT, 1995, p. 246.

5. Short observations on a printed paper, in Locke, 1991, vol. II, p. 350 : John Locke explique ici qu'une dévaluation réduirait les revenus du roi, les rentes, l'ensemble des dettes, en même temps qu'elle perturberait le commerce. 
techniques qui sont celles du monnayage et de la fixation du taux d'intérêt prend son sens dans le cadre plus général d'une analyse de la propriété, du travail, de l'enrichissement individuel et national, du commerce et des relations entre nations. Il faut d'ailleurs rappeler que c'est à ce niveau général que se situent les controverses de l'époque sur les questions monétaires. Les principaux adversaires de Locke dans ce débat, sir Josiah Child d'abord (sur la question du taux d'intérêt), puis William Lowndes et Thomas Neale (concernant le projet de dévaluation), ont en commun avec lui de défendre une conception structurée de la richesse et de proposer une analyse internationale en termes de balance commerciale, conformément à l'approche mercantiliste en vigueur. Ils occupent tous, également, des fonctions politiques de premier plan (Child dirige l'East India Company, Lowndes est secrétaire au Trésor et Neale est maître de l'hôtel des Monnaies) qui confèrent à leurs analyses des enjeux concrets immédiats. De même, les analyses de Locke, qui lui sont demandées au titre d'expert, pèseront sur la décision du Parlement d'entreprendre en 1695 la refrappe de la monnaie d'argent en circulation.

Locke est en ce sens un représentant typique des théoriciens économiques de son temps. Il l'est moins en ce qui concerne les considérations théoriques, poussées chez lui à un plus haut niveau d'exigence philosophique et politique, qu'atteste clairement son analyse de la monnaie et de la richesse. Il est donc intéressant d'essayer de discerner ce qui fait l'originalité de son approche sur fond de problématiques partagées et de questions, au moins partiellement, communes. Ces questions portent notamment sur le rapport entre monnaie et richesse. On sait qu'Adam Smith attribuera au mercantilisme une unité doctrinale fondée sur la confusion entre richesse et monnaie métallique. Ce jugement a été depuis longtemps remis en question. Il n'en reste pas moins vrai que tout le XVII siècle se pose le problème du rapport entre l'activité économique nationale et la quantité de monnaie en circulation, même si les réponses diffèrent considérablement d'un auteur à l'autre.

Locke traite cette question d'une façon qui est souvent présentée comme paradoxale, en ce qu'elle tente d'associer à l'objectif d'une balance commerciale excédentaire ${ }^{6}$ l'affirmation qu'une hausse des prix résulte de l'accroissement de la quantité de monnaie en circulation. Mais s'agit-il vraiment d'une contradiction? Locke considère bien que le niveau des prix est en relation directe avec la quantité de monnaie en circulation ${ }^{7}$. Mais la

6. Propositions sent to the lords justices, in Locke, 1991, vol. II, p. 378 : « An overbalance of trade is when the quantity of commoditys, which we send to any country, do more than pay for those we bring home of theirs. For then the overplus is brought home in money. »

7. Cette hypothèse, dont on trouve les premiers linéaments dès l'Antiquité, est formulée par Copernic et surtout par Bodin, au cours de la controverse qui l'oppose à Malestroit concernant les effets de l'afflux des métaux précieux sur la hausse des prix, voir GugGENHEIM, 1978, 
quantité de monnaie est à distinguer de la richesse de la nation et n'est finalement que l'instrument de sa circulation. Pour être bien compris, le problème de la nature et de la fonction de la monnaie qui en découle doit être relié directement à l'angle d'approche choisi. Instrument de circulation et réserve de valeur, elle est aussi lien social et moyen d'intervention politique : loin de réduire les questions économiques à leur seul aspect monétaire, Locke pense la monnaie comme le lieu théorique d'intégration de toutes les dimensions de l'analyse et comme le lieu pratique de leur interaction maîtrisée.

Au premier abord, Locke semble être un tenant de la monnaie-signe, simple représentation conventionnelle, obtenue par le consentement général, des valeurs des diverses marchandises en circulation : "L'humanité a consenti à attribuer une valeur imaginaire à l'or et à l'argent, en raison de leur rareté, de leur durabilité et de la difficulté à les contrefaire ${ }^{8}$. » Pourtant, il affirme bien par là même que cette valeur « imaginaire » n'est pas le moins du monde arbitraire ${ }^{9}$ : les qualités des métaux précieux motivent leur choix en tant qu'instruments d'échange, augmentant le volume du commerce et permettant l'accumulation de la richesse. Dans le débat qui oppose la notion de monnaie-signe à celle de monnaie-marchandise, la monnaie lockienne est, si l'on veut, une marchandise-signe, soumise comme les autres biens à la loi de l'offre et de la demande, mais dotée, de par sa fonction propre, de propriétés spécifiques, qu'aucune autre marchandise ne saurait posséder. Il y a assurément une difficulté de l'analyse lockienne sur ce point : mais elle consiste moins en une contradiction inaperçue qu'en la détermination problématique d'une double nature de la monnaie :

« [L'argent] sert à la fois comme jetons et comme gages, et [...] donc il véhicule avec lui un compte uniforme, et la garantie que celui qui le reçoit, aura en contrepartie à nouveau la même valeur, en autres choses qu'il désire, quand il lui plaît. Il remplit l'un par son empreinte et sa dénomination; l'autre par sa valeur intrinsèque, qui est sa quantité ${ }^{10}$.»

\footnotetext{
$1^{\text {re }}$ part., chap. II. Au $\mathrm{xx}^{\mathrm{e}}$ siècle, Irving Fischer en proposera une formulation algébrique, l'« équation générale des transactions », voir RuFFinI, 1996, p. 60.

8. Quelques considérations sur les conséquences de l'abaissement de l'intérêt et de l'élévation de la valeur de la monnaie, in LocKE, 1983, p. 70. Cette traduction ne concerne que les pages 209 à 236 de l'édition anglaise du texte de Locke, voir Some considerations, in Locke, 1991 , vol. I. On citera à chaque fois que c'est possible cette traduction, en indiquant les références correspondantes dans l'édition anglaise. Ici, Some considerations, in Locke, 1991, vol. I, p. 233.

9. De ce point de vue, cette valeur imaginaire de la monnaie n'a pas à être « contrebalancée » par la durabilité des métaux précieux, comme l'affirme Рососк, 1997, p. 458.

10. Locke, 1983a, p. 70; 1991, vol. I, p. 233.
} 
La monnaie est une fonction, dont l'or est le support matériel : la distinction des deux doit surtout s'accompagner de l'explication de leur connexion privilégiée, car Locke est loin d'être partisan de la circulation d'une monnaie de papier, des lettres de change ${ }^{11}$ et il préconise la refrappe de ce qu'on nomme à l'époque la «mauvaise monnaie », c'est-à-dire des pièces rognées dont la valeur nominale est supérieure à la valeur marchande. Il lui faut donc donner une explication de la formation de la valeur de la monnaie métallique, qui tienne à la fois compte de sa fonction et de sa nature de marchandise. Et c'est ce à quoi s'emploient les divers essais économiques.

La monnaie doit d'abord être décrite comme une marchandise : elle présente une valeur d'échange, mais celle-ci se fonde sur une valeur préexistante, la «valeur intrinsèque », dont Locke emprunte l'expression à la scolastique $^{12}$. Cette valeur intrinsèque, de nature substantielle, doit pourtant présenter également une dimension conventionnelle, pour pouvoir devenir le support d'un système de signes dont on peut constater empiriquement le caractère variable. De plus, les phénomènes économiques relèvent pour Locke d'une «prudence» pratique, qui concerne les modes mixtes, non pas les substances ${ }^{13}$. Et l'on s'aperçoit alors que la double définition lockienne de l'or comme substance et de l'or comme monnaie s'effectue conjointement dans les écrits économiques et dans l'Essai philosophique concernant l'entendement humain: cette distinction est cruciale puisque c'est elle qui permet d'attribuer à l'or sa double nature de marchandise et d'instrument d'échange. Mais avant d'examiner l'Essai, il convient de préciser d'abord la fonction monétaire de l'or et de l'argent.

L'or, en tant que marchandise, possède une valeur qui est définie, d'une part, par le travail nécessaire à son extraction, d'autre part, par le rapport entre l'offre et la demande concernant ce bien précis. Et c'est en ce point que les « confusions » de Locke se révèlent être des thèses philosophiques essentielles : l'offre et la demande définissent moins l'espace d'un marché libéré de toute contrainte et fonctionnant selon ses règles propres, que l'utilité spécifique d'un bien, « sa capacité à fournir les nécessités ou à procurer les commodités de la vie humaine ${ }^{14} \gg$. La valeur d'une marchandise est

11. Quelques considérations, in Locke, 1991, vol. I, p. 68 : «Rien ne paiera les dettes que l'argent ou ce qui a valeur d'argent, ce que trois ou quatre lignes écrites sur un papier ne peuvent pas être. »

12. Cette notion de valeur intrinsèque permet avant tout à Locke de souligner le lien indestructible qui existe entre l'économie et la nature, en tant qu'elle est mise par Dieu à notre disposition pour la réalisation de nos finalités propres.

13. LOCKE, 1983 b, p. 602.

14. Some considerations of the consequences of the lowering of interest and raising the value of money, in Locke, 1991, vol. I, p. 258 : "Its fitness to supply the necessities or serve the conveniencies of human life. " 
d'emblée de nature relationnelle, et se définit comme une proportion entre la quantité d'un bien et sa demande : Locke est loin d'une définition de la valeur-travail et il inscrit dans la nature de la marchandise son caractère social ${ }^{15}$. L'utilité est une demande sociale qui détermine le niveau des besoins et fournit son cadre à la recherche individuelle du bien-être. Distincte du prix naturel de Smith, déterminé par la quantité de travail commandé, la valeur intrinsèque renvoie immédiatement à l'ensemble d'une société, à ses habitudes de consommation et surtout à ses valeurs éthiques et à sa définition du bonheur, tant individuel que collectif. La valeur d'usage inclut la valeur d'échange plus qu'elle ne la détermine ${ }^{16}$ et la monnaie est un lien social qui assure la répartition de la richesse collective.

De ce point de vue, la monnaie fait face à une demande qui, selon Locke, demeure constante. Les variations de sa valeur ne peuvent donc provenir que de la quantité de monnaie en circulation, qui détermine à son tour le niveau des prix, compte tenu de sa vitesse de circulation, de l'état du commerce, des dettes ${ }^{17}$. Il importe donc que cette quantité de monnaie soit correctement définie : si la production d'or augmente, l'unité monétaire voit sa valeur diminuer et on assiste à un mouvement de hausse des prix. Cette proposition constitue ce qu'on nomme le théorème quantitatif, qui établit donc une stricte proportionnalité entre la quantité de monnaie et le niveau général des prix. En conséquence de cet énoncé, une augmentation du stock de monnaie n'est nullement assimilable à une augmentation de richesse. Pourtant, Locke maintient l'idée mercantiliste selon laquelle il est bénéfique à une nation de capter plus d'or et d'argent que les autres. Cette affirmation semble au premier abord incompatible avec le théorème quantitatif. Pourtant, Locke prend soin de noter que ce n'est pas la quantité de métal précieux qui fait la richesse, mais la quantité de métal monnayé qui constitue à la fois le signe et l'instrument de la prospérité économique. La preuve en est que « les pays qui sont les plus pourvus [de mines] par la nature sont pauvres ${ }^{18} \gg$. Locke ne construit pas une théorie de la valeur, mais une théorie de la richesse qui articule en permanence économie marchande et puis-

15. Voir Propositions sent to the lords justices, in LocKe, 1991, vol. II, p. 374 : «The intrinsick value of silver considerd as money is that estimate which for its fitnesse, common consent has placed on silver in making the universal exchange or barter for all other commoditys and by that it is the instrument of commerce."

16. Voir LocKe, 1983a, p. 70; 1991, vol. I, p. 233-234 : « La valeur intrinsèque concernant ces métaux institués pour le troc commun n'est rien d'autre que la quantité que les hommes en donnent ou en reçoivent. »

17. Hyde Kelly, 1991, p. 72.

18. Locke, 1983a, p. 56. Voir aussi Further considerations concerning raising the value of money, in Locke, 1991, vol. II, p. 419 : «Money also is necessary to us, in a certain proportion to the plenty of it amongst our neighbours. » 
sance politique, phénomènes monétaires et souveraineté : «La fin principale du commerce est les richesses et la puissance ${ }^{19}$. »

L'or n'est pas une richesse naturelle mais son rôle est le résultat de la circulation des marchandises, après qu'il en ait été historiquement la condition $^{20}$. On peut alors comprendre qu'il soit important pour une nation d'en posséder une proportion telle que sa position dans le commerce mondial se trouve par là même avantagée. La complexité de l'analyse lockienne résulte de la présentation de la monnaie à la fois comme signe de la puissance économique et politique, et comme équivalent de la richesse. La première se définit comme une quantité de force politique et la seconde comme une proportion sur le terrain de l'échange. D'où la curieuse solution de Locke : «Les richesses ne consistent pas dans le fait d'avoir plus d'or et d'argent, mais d'en avoir proportionnellement plus que le reste du monde $^{21}$.» On peut noter qu'une telle proposition ignore la production au profit de la circulation, mais il importe surtout de considérer qu'elle s'éclaire du point de vue du commerce international qui est celui de Locke $^{22}$, et qu'il tente de concilier avec l'étude de la richesse nationale, de sa formation et de ses transferts. Même s'il méconnaît le phénomène de l'inflation et n'attribue la perte du pouvoir d'achat de la monnaie qu'au seul rognage des pièces ${ }^{23}$, Locke s'efforce de penser les phénomènes monétaires à la fois dans leur spécificité et dans leur dépendance par rapport à des réalités économiques et politiques globales, qui font du monde dans sa totalité le terrain d'une lutte acharnée ${ }^{24}$.

En ce sens, si les variations de la quantité de monnaie n'influent pas sur les prix relatifs nationaux, elles sont de la première importance dès lors qu'on envisage leur effet sur les prix des biens importés et des biens exportés. Locke envisage par hypothèse une diminution de moitié de la quantité de monnaie en circulation, diminution qui hausserait les prix des biens intérieurs et diminuerait ceux des biens étrangers, provoquant aussitôt l'émigration de ceux qui peuvent espérer des revenus plus élevés hors d'Angle-

19. "Trade», in Locke, 1991, vol. II, p. 485 : «The cheif end of trade is riches and power. » Shaftesbury, cité in Hill et Postan, 1977, p. 370, déclare pour sa part : "C'est le commerce et le commerce seul qui entraîne après soi cette richesse et cette puissance sur mer qui ne sauraient s'acquérir par aucun autre moyen. »

20. Dans le chapitre v du Second traité, Locke présente une histoire de l'apparition de la monnaie empruntée à Aristote. Cette analyse ne l'empêche pas de distinguer la fonction initiale de la monnaie de son rôle ultérieur de moteur de la circulation.

21. LocKe, 1983a, p. 57; 1991, vol. I, p. 222.

22. BRAudel, 1979 , p. 439 et 474 : Fernand Braudel signale qu'à cette époque, « la distribution [...] est le vrai secteur du profit » et que, même si son volume est souvent inférieur à celui du commerce intérieur « le commerce au loin a, sans doute, tenu le premier rôle dans la genèse du capitalisme marchand, il en fut longtemps l'ossature ».

23. VILAR, 1974, p. 271.

24. STEINER, 1992, p. 111-114. 
terre $^{25}$. L'analyse monétaire n'est donc jamais coupée de sa dimension politique et sociale et il s'instaure entre les deux niveaux un système de relations causales réciproques. C'est ainsi que le niveau des prix n'est pas seulement à envisager par rapport à la quantité nationale de monnaie, mais aussi par rapport aux prix en vigueur dans les nations voisines avec lesquelles existent des relations commerciales: il modifie les termes de l'échange et sert d'instrument à disposition d'une politique économique qui prône l'exportation des biens manufacturés ainsi que la limitation des importations de matières premières. L'angle d'approche original construit par Locke contribue à faire de la monnaie le foyer théorique d'une analyse globale qui reconnaît aux phénomènes monétaires leur spécificité mais non leur autonomie.

Tout concourt donc à faire de la monnaie lockienne une réalité complexe, dont la nature se détermine à différents niveaux d'analyse ${ }^{26}$ et qui les met en relation. Sa double nature de métal précieux et de signe conventionnel pose des problèmes qui sont à la fois de nature économique, en tant qu'ils concernent la politique internationale et la fixation du taux d'intérêt, et de nature philosophique, en ce que l'articulation des deux niveaux requiert une explication précise. En effet, la monnaie se définissant également comme marchandise, il faut déterminer ce qui lui confère sa fonction privilégiée d'instrument de la circulation et de mesure de la valeur des autres marchandises. Le problème se déplace sur le terrain d'une analyse de la représentation, située au cœur même de la philosophie lockienne et qui doit s'aider de la distinction entre substance, mode mixte et relation, telle que l'élabore l'Essai philosophique concernant l'entendement humain. Concernant ce dernier point, il est vrai qu'aucun chapitre de l'Essai ne porte sur la monnaie en tant que telle. Pourtant, on ne peut qu'être frappé par la fréquence du recours à l'exemple de l'or dans cette œuvre. Plusieurs éléments permettent effectivement de rattacher les analyses monétaires de Locke aux considérations philosophiques de l'Essai.

Tout d'abord, comme l'a noté Patrick Hyde Kelly, Locke emploie fréquemment le terme de «notion» dans ses écrits économiques. Or ce terme

25. Some considerations, in Locke, 1991, vol. I, p. 266.

26. À la même époque, des théoriciens de l'économie comme Josiah Child ou Dudley North s'opposeront effectivement à l'intervention politique, par hostilité à l'égard des restrictions aux exportations qui gênent les grandes compagnies. North, cité in Pribram, 1986, p. 69, va jusqu'à écrire que « le flux et reflux de la monnaie s'organise sans aucune aide des politiciens ». 
renvoie aux idées complexes considérées comme modes mixtes ${ }^{27}$. Ranger l'économie du côté de l'analyse des modes mixtes, c'est lui conférer le rang d'une pratique prudentielle, qui porte non pas sur des substances, mais sur « certaines combinaisons d'idées simples, qu'on ne regarde pas comme des marques caractéristiques d'aucun être qui ait une existence fixe, mais comme des idées détachées et indépendantes, que l'esprit joint ensemble ${ }^{28} \gg$. Ces combinaisons, au lieu d'être dictées par les choses, sont construites par l'esprit qui peut donc les connaître pleinement et éventuellement agir sur elles : le propre d'une notion est bien d'être «plutôt fondée sur les pensées des hommes que sur la nature même des choses ${ }^{29}$ ». C'est parce que le taux d'intérêt est une notion qu'on peut le modifier par décision. Pourtant, un problème délicat surgit : si le taux d'intérêt est une notion plastique, elle n'existe que par rapport à un autre mode mixte, la monnaie, qui est le fruit du consentement mais aussi un métal monnayé, dont la valeur ne saurait être fixée de façon arbitraire. On retrouve donc, à ce niveau ontologique et épistémologique, le paradoxe économique d'une convention fondée en nature, d'une monnaie qui est à la fois signe et métal, mode mixte d'origine sociale et substance naturelle. Pour cette raison, Locke demeure inclassable et oscille entre métallisme et cartalisme ${ }^{30}$, en construisant une théorie de la monnaie comme chose dotée d'une fonction de représentation, théorie que Constantine George Caffentzis a qualifiée de « sémantique ${ }^{31}$ ».

Toute représentation est le résultat d'une genèse. Il convient donc de partir d'abord de l'or comme substance pour envisager la construction sur cette base de la notion de monnaie. «Les idées des substances, écrit Locke, sont certaines combinaisons d'idées simples, qu'on suppose représenter des choses particulières et distinctes, subsistant par elles-mêmes ${ }^{32}$. » L'idée est, dans ce cas, une copie qui renvoie à un archétype existant indépendamment de notre connaissance. La connaissance des substances relève de la philosophie de la nature, mais elle ne peut être ni directe ni complète : il est alors nécessaire de distinguer nettement l'essence réelle de l'essence nominale. L'essence réelle demeure inaccessible, simple support des qualités que nous repérons empiriquement et que nous sommes portés à attribuer aux choses mêmes :

27. Hyde Kelly, 1991, p. 93.

28. LOCKE, 1983b, p. 224.

29. LOCKE, 1983b, p. 225.

30. C'est ce que note SCHUMPeter, 1983, p. 407. Le cartalisme est l'affirmation que la monnaie est un ticket ou un bon, et non une marchandise.

31. CAFFentzis, 1989, p. 84 : «The only hope for the continuation of the world trade was that silver (and/or gold) continued to be an element of the idea of money, since this substance would provide a supra-social point of agreement necessary for the coordination of a huge variety of individual minds expressing themselves in a cacophony of tongues. »

32. LOCKE, 1983b, p. 119. 
«Par exemple, l'essence nominale de l'or, c'est cette idée complexe que le mot or signifie, comme vous diriez un corps jaune, d'une certaine pesanteur, malléable, fusible, et fixe. Mais l'essence réelle, c'est la constitution des parties insensibles de ce corps, de laquelle ces qualités et toutes les autres propriétés de l'or dépendent. Il est aisé de voir d'un coup d'œil combien ces deux choses sont différentes, quoiqu'on leur donne à toutes deux le nom d'essence ${ }^{33}$.»

Si la propension de notre esprit est d'affirmer que les substances sont bien telles que nous les définissons, cette opération même de définition intercale la dénomination, ou l'essence nominale, entre la substance et nous. La reconnaissance de cette opération de construction incite à davantage de prudence quant à la portée effective de nos conclusions :

« À l'égard des essences réelles des substances, nous supposons seulement leur existence sans connaître précisément ce qu'elles sont. Mais ce qui les lie toujours à certaines espèces, c'est l'essence nominale dont on suppose qu'elles sont la cause et le fondement ${ }^{34}$. »

L'or est donc avant tout la dénomination d'une espèce fondée sur la sélection de certaines propriétés qui donnent au mot sa signification précise. Il est une représentation que nous avons tendance à prendre pour une chose, parfois à nos dépens, puisque nous nous fions le plus souvent à la figure et à la couleur pour former nos idées complexes des substances :

« Ainsi là où nous trouvons la couleur de l'or, nous sommes portés à nous figurer que toutes les autres qualités comprises dans notre idée complexe y sont aussi, de sorte que nous prenons communément des deux qualités qui se présentent d'abord à nous, la figure et la couleur, pour des idées si propres à désigner différentes espèces, que voyant un bon tableau nous disons aussitôt, c'est un lion, c'est une rose, c'est une coupe d'or ou d'argent ${ }^{35}$.»

On ne saurait mieux dire que l'or est la copie idéelle ${ }^{36}$ d'une substance et que le faussaire est à cet égard meilleur philosophe que le fétichiste. La monnaie est donc le résultat perfectionné et tardif de ce même processus de représentation, qui englobe sous un même nom un nombre plus ou moins élevé de choses, en fonction des propriétés qui ont été retenues :

«La même commodité qui a porté les hommes à désigner par un seul nom les diverses pièces de cette matière jaune qui vient de la Guinée ou du Pérou, les

33. LOCKE, 1983b, p. 353-354.

34. LOCKE, 1983b, p. 356.

35. LOCKE, 1983b, p. 368.

36. LOCKE, 1983b, p. 291, parle de «peinture mentale». 
engage aussi à inventer un seul nom qui puisse comprendre l'or, l'argent et quelques autres corps de ces différentes sortes ${ }^{37}$. »

La notion de métal qui en résulte se fonde sur une convention, qui renvoie à une utilité et à un usage communs, qu'il s'agisse du métal utilitaire ou du métal monnayable. De la même façon, l' « espèce » monétaire est une «portion de matière », "considérée comme rangée sous la dénomination d'une certaine idée abstraite ${ }^{38}$. L'important est de considérer que «lorsque les hommes forment leurs idées génériques des substances, ils ne suivent pas exactement les modèles qui leur sont proposés par la nature $^{39} \gg$ : les genres et les espèces procèdent d'une opération humaine de découpe du réel, qui leur confère d'emblée un caractère conventionnel. La monnaie peut alors être considérée comme le résultat exemplaire d'une telle procédure de fixation des significations et qui commence obligatoirement par l'identification certaine des métaux précieux qui lui servent de support matériel.

Il en résulte que les idées d'or sont variables, en fonction des propriétés connues et sélectionnées par ceux qui emploient ce terme. Locke montre que l'origine adamite des noms n'est que le commencement singulier d'un processus qui se poursuit et se modifie sans cesse : Adam retient de l'or qu'il est «un corps dur, brillant, jaune et fort pesant ${ }^{40}$ » et le nomme zahab. Mais en multipliant les expériences sur ce métal, il sera en mesure d'allonger presque sans fin cette liste. Le danger qui en résulte est que chacun donne aux mots un sens différent et soit porté à penser qu'ils désignent immédiatement les essences réelles. Cette substantification illusoire du langage fait naître le risque de son éclatement en idiomes incommunicables. Face à celui-ci, il faut rappeler que c'est l'usage social de la langue qui fixe les significations communes. C'est pourquoi « cette affirmation, tout or est fixe, ne contient autre chose que la signification du terme d'or ${ }^{41} »$. De la même manière, le grand avantage des dénominations monétaires, et l'utilisation de l'unité shilling pour énoncer les prix, précise Locke, est qu'elle est une mesure dont « les idées, par leur usage constant, se sont imprimées dans l'esprit de tout Anglais ${ }^{42} »$. La monnaie est un signe particulièrement

37. LOCKE, 1983b, p. 371.

38. LOCKE, 1983b, p. 356.

39. LOCKE, 1983b, p. 371.

40. LOCKE, 1983b, p. 379.

41. LOCKe, 1983b, p. 379-380.

42. Some considerations, in Locke, 1991, vol. I, p. 249 : "These are measures whose ideas by constant use are setled in every english man's mind. »Voir Short observations, in LOCKE, 1991, vol. II, p. 354 : "To avoid fallacies, I desire to be understood when I use the word silver alone, to mean nothing but silver, and do lay aside the consideration of baser metal that may be mixed with it [...]. The silver being the measure of commerce, 'tis the quantity of silver that is in every piece he receives, and not the denomination of it which the merchants look after, and values it by. » 
fiable parce qu'il est matériellement inscrit sur la chose même qu'il désigne, une quantité donnée d'or ou d'argent fins.

L'idée de l'or en tant que substance est donc une combinaison d'idées simples qui correspondent aux qualités sensibles que nous avons identifiées et retenues dans notre définition de ce terme. Une telle connaissance est certes incomplète, mais suffisante : «Ainsi nous pouvons, par le secours des sens, connaître et distinguer les choses, les examiner autant qu'il est nécessaire pour les appliquer à notre usage, et les employer en différentes manières à nos besoins dans cette vie ${ }^{43}$. Dieu a fait en sorte que notre intelligence convienne à la connaissance de nos devoirs et à la satisfaction des nécessités de la vie. Et toute la réflexion lockienne sur le statut et la nature de la connaissance ouvre à la définition du comportement éthique et pratique propre à l'homme. En ce sens, le choix de l'or comme exemple récurrent tout au long de l'Essai ne semble pas relever du hasard: une connaissance sensible trop perfectionnée, par exemple une vision quasi microscopique de la matière serait un handicap pour celui qui ne pourrait de toute façon pas «se servir d'une vue si perçante pour aller au marché ou à la bourse ${ }^{44} \gg$. Les idées complexes que nous avons des substances matérielles visent moins à l'édification d'une science de la nature inutile qu'à l'organisation rigoureuse d'un savoir pratique conforme à notre destination. La connaissance de toute façon impossible de l'or en tant que substance doit céder la place à la construction d'une idée suffisante pour notre usage. En l'occurrence, cet usage est double : l'or est un métal qui sert à la fabrication d'objets divers, il est aussi un instrument d'échange, le substrat de la fonction monétaire. Dans ce second emploi, il devient le support matériel d'une convention, d'un système de signes qui constitue la monnaie proprement dite.

On se trouve alors dans le cas d'une construction de niveau deux : l'idée complexe d'or donne lieu à une nouvelle dénomination, de type monétaire, qui crée un nouveau type d'espèce à partir d'une convention supplémentaire, qui limite le nombre des qualités secondes retenues au poids et à la pureté du métal échangé. Ce cas n'est pas développé dans l'Essai, mais on y trouve tous les éléments conceptuels qui permettent de comprendre le traitement lockien des phénomènes monétaires dans les essais économiques. En effet, Locke considère la monnaie comme un mode mixte, dans la mesure où sa dénomination conventionnelle l'arrache à tout modèle dont elle ne serait que la copie. La monnaie est à elle-même son propre archétype et peut être définie par pure convention : la valeur d'une frappe est toujours vraie en ce qu'elle définit des unités de comptes qui ne lui

43. LOCKE, 1983b, p. 236.

44. Locke, 1983b, p. 237. 
préexistent pas $^{45}$. Mais on aperçoit aussitôt la difficulté : la frappe peut ne pas correspondre à la quantité de métal précieux qu'elle est censée désigner. La monnaie est un mode mixte qui renvoie à une substance, une convention qui fait fond sur une nature et dont se révèle le caractère paradoxal en cas de crise monétaire ou de fraude ${ }^{46}$.

Face à cette situation, il ne s'agit donc nullement de développer une science de l'or-substance mais une théorie de l'or-monnaie, directement en prise sur la pratique des échanges et l'organisation politique de la société. Sachant que l'or est un mode mixte constitué d'un agrégat de qualités secondes qui sont autant de puissances de sa substance, il est possible de définir la monnaie par simplification et épuration de notre définition première : si l'on ramène le mode «métal précieux » à deux idées simples, qui sont la pureté et le poids, on le réduit à une relation ${ }^{47}$, aisément manipulable par l'esprit et garante de l'adéquation entre la représentation et ce qui est représenté : « Nos idées simples sont toutes réelles et conviennent avec la réalité des choses ${ }^{48}$. »

Les dénominations monétaires renvoient à ces deux relations, pureté et quantité, qui fondent leur parfaite fiabilité : «La relation est un moyen de comparer ou de considérer deux choses ensemble, en donnant à l'une ou à toutes les deux quelque nom tiré de cette comparaison, et quelquefois en désignant la relation même par un nom particulier ${ }^{49}$. » Le lien à la substance n'a pas à être exploré plus avant, il suffit que la dénomination monétaire instaure un parallélisme constant et fiable avec le métal précieux considéré comme chose : «La réalité de ces idées [consiste] dans cette continuelle et variable correspondance qu'elles ont avec les constitutions distinctes des êtres réels ${ }^{50}$. $\gg$ On comprend alors que Locke soit hostile au bimétallisme or-argent qui, en admettant l'existence de deux étalons dis-

45. Locke, 1983b, p. 309 : «Lorsque nous jugeons de la vérité de nos idées par la conformité qu'elles ont avec celles qui se trouvent dans l'esprit des autres hommes, et qu'ils désignent communément par le même nom, il n'y en a point qui ne puissent être fausses dans ce sens là. »

46. Locke, 1983b, p. 511, envisage dans l'Essai l'invention individuelle d'une monnaie imaginaire qui ne pourrait par conséquent avoir aucun usage : l'inventeur «peut sans doute compter fort exactement, et assembler une grosse somme [...] sans être pourtant plus riche d'une pite et sans savoir même combien vaut un écu, une livre ou un sou, mais seulement que l'un est contenu trois fois dans l'autre, et contient l'autre vingt fois, ce qu'un homme peut faire aussi dans la signification des mots en leur donnant plus ou moins d'étendue considérés l'un par rapport à l'autre ». Voir, par ailleurs, Short observations, in LocKE, 1991, vol. II, p. 346 : "The stamp neither does nor can take away any of the intrinsick value of the silver, and therefore an ounce of coin'd standard silver, must necessarily be of equal value to an ounce of coin'd standard silver. "

47. Locke, 1983b, p. 250 : les relations sont les idées que «l'esprit forme de la comparaison [des] choses entre elles ».

48. Locke, 1983b, p. 296.

49. LOCKE, 1983b, p. 253.

50. Locke, 1983b, p. 296. 
tincts, réintroduit la question de l'estimation réciproque variable de ces deux métaux, et qu'il répète à l'envi qu' « une égale quantité d'argent est toujours d'une même valeur qu'une égale quantité d'argent. C'est ce que le sens commun aussi bien que le marché nous enseignent ${ }^{51}{ }^{\prime}$. La relation monétaire est alors l'occasion de la confiance des échangistes et l'objet d'une certitude suffisante pour devenir l'instrument idéal du commerce.

La monnaie a bien le statut d'un langage dont les conventions collectives ont valeur de norme, mais les signes sont alors apposés sur les choses matérielles elles-mêmes, réduisant à zéro les risques d'erreur concernant la valeur représentative de ces mêmes signes. En premier lieu, le caractère intersubjectif des conventions, et par excellence de la convention monétaire, évite tout risque de contestation : tout homme est apte à distinguer les diverses pièces de monnaie et à connaître parfaitement leur essence nominale, ou leur valeur (qu'on qualifie d'ailleurs elle aussi de nominale). En second lieu, cette fixation collectivement admise de la valeur de la monnaie permet d'espérer une connaissance économique bien plus avancée que ne saurait l'être celle de la substance or. C'est en ce sens seulement qu'on peut parler de l'émergence d'une science économique chez Locke, et qui a le même statut que la morale, dont il pense à l'époque de la rédaction de l'Essai qu'elle est «capable de démonstration ${ }^{52}$ », au même titre que les mathématiques.

En ce qui concerne l'économie, la définition d'un standard monétaire permet la création volontaire et consensuelle de la base de l'édifice monétaire, qui peut alors faire l'objet d'une connaissance certaine : l'étalon est l'archétype d'un mode mixte dont l'idée est la représentation adéquate. C'est en ce sens qu'il faut comprendre la défense farouche par Locke du caractère intangible de l'étalon. Il fournit à la théorie autant qu'à la pratique son point d'ancrage stable, le réfèrent de toute politique économique. Locke le dit et le répète : «Le standard devrait être, une fois fixé, inviolable et immuable à perpétuité ${ }^{53}$. » Cela ne signifie nullement que l'ensemble de l'analyse économique soit une science démonstrative, car s'ouvrant sur les décisions politiques, les comportements collectifs, les choix individuels, les circonstances historiques, elle présente le caractère d'un savoir social qui admet que l'essence réelle de l'homme reste hors de sa portée. Les concepts de l'économie lockienne sont descriptifs et s'efforcent d'être prescriptifs, c'est pourquoi la tonalité pragmatique des

51. Further considerations, in LocKE, 1991, vol. II, p. $411:$ « Hence it is evident, that an equal quantity of silver is always of equal value to an equal quantity of silver. This common sense, as well as the market teaches us. »

52. LOCKe, 1991, vol. II, p. 419.

53. Some considerations, in LoCKE, 1991, vol. I, p. 329 : «The standard once thus settle, should be inviolably an immutably kept to perpetuity. » 
écrits économiques est moins la preuve de leur caractère subalterne que la marque du statut spécifique du savoir qu'ils développent. Et à la différence de la morale, la raison ne suffit pas ici à nous indiquer les actions conformes à notre destination ${ }^{54}$ : le recours à l'expérience semble indispensable pour valider les choix économiques. Ancrée dans une convention, le standard métallique fixe, l'économie peut s'ouvrir sur la complexité des comportements sociaux, sans perdre pour autant de vue le point cardinal de ce principe objectif, en ramenant toujours la monnaie à une relation quantifiable par l'intermédiaire de dénominations invariables. C'est pourquoi l'analyse économique lockienne, tout en présentant d'emblée une forte originalité, reste liée à la définition baconienne d'une histoire naturelle, qui s'attache à collecter de façon systématique des données empiriques ${ }^{55}$.

La construction d'un savoir économique débouchant sur une pratique réglée est donc une opération délicate. Par exemple, les modalités de fixation de la valeur de la monnaie sont complexes et ne sont pas à la portée des décisions individuelles, pas plus qu'à celle de la seule volonté gouvernementale ${ }^{56}$. Pour une part même, l'or continue d'être une marchandise recherchée en tant que substance matérielle et dont la valeur est fixée indépendamment de toute décision, par le jeu de l'offre et de la demande et par le travail ${ }^{57}$. À tenir liées ces deux affirmations, Locke se trouve face à une difficulté théorique immense, qui sera celle de l'ensemble des théories monétaires après lui, jusqu'à Marx inclus. D'où provient la valeur de la monnaie, et comment se détermine son prix ? On peut considérer que ses principaux énoncés économiques se distribuent sur le double axe de la nature et de la convention ainsi dessiné et consistent, d'une part dans sa théorie du taux d'intérêt, d'autre part dans sa théorie du monnayage, qu'on examinera successivement. De ce point de vue, l'étude lockienne de la représentation se trouve bien mise au service de la construction d'une analyse monétaire spéciale, qui en reprend mais surtout en complète et en prolonge les considérations générales.

54. Quelques considérations, in LocKe, 1983a, p. 72 ; 1991, vol. I, p. 235 : Locke reconnaît qu' « il est impossible d'évaluer exactement la quantité d'argent nécessaire dans le négoce » en raison du nombre des paramètres à prendre en considération, et en particulier du rôle de la vitesse de circulation de la monnaie. Par ailleurs, il propose aux partisans de la dévaluation de tester empiriquement leur hypothèse, voir Hyde Kelly, 1991, p. 94-95.

55. Locke est lié, à travers Boyle et Petty, à un courant d'inspiration baconienne, qui joint à l'analyse systématique des données disponibles, notamment dans le domaine de l'agriculture, une ambition réformatrice, voir Wood, 1984, p. 20-35.

56. CAFFENTZIS, 1989, p. 163, l'a bien montré : «The accumulation process and the state are preconditioned on the monetary system; should it break down, so will they. Mistakes with money are fatal. "

57. LocKe, 1994, p. 37, n'explique pas comment se combinent ces deux sources de la valeur : «L'or et l'argent, qui sont si peu utiles à notre vie au regard de la nourriture, du vêtement et des transports, ne tiennent leur valeur que du consentement des hommes, lequel se règle en grande partie sur le travail. » 
Concernant d'abord le loyer de l'argent, il faut rappeler que c'est l'usage qui définit les modes mixtes : Locke milite pour un taux «naturel » de l'intérêt, fixé par accord mutuel des échangistes sur la base de l'état du marché. On connaît le début des Quelques considérations : «La première chose à considérer est de savoir si le prix du loyer de l'argent peut être réglé par la loi. Et à mon avis, d'une manière générale, on peut dire qu'il est évident qu'il ne peut l'être ${ }^{58}$. » Mais Locke préconise la fixation d'un taux national de l'intérêt, « raisonnable », empêchant les prêteurs de profiter «de l'ignorance ou de la nécessité des emprunteurs » ${ }^{59}$. La politique monétaire doit suivre l'évolution naturelle du taux de façon à en faire à la fois le résultat d'un équilibre mécanique et une convention collective qui place tout le monde à égalité devant le crédit. Cette mesure est moins inspirée par un esprit libéral avant la lettre que par l'analyse de la monnaie comme convention, soumise doublement aux lois du marché et à la décision des hommes.

Locke lutte explicitement contre le monopole des banquiers, il se préoccupe assurément aussi des conditions de remboursement de la dette publique de l'Angleterre, mais sa conception de l'intérêt ne saurait être coupée d'une analyse plus globale de l'échange, de ses finalités et de ses conditions, individuelles et sociales. Il s'efforce en effet de définir le rôle de l'autorité en matière monétaire : si l'autorité publique se charge de la frappe de la monnaie, c'est bien le consentement commun qui décide que l'or et l'argent sont monnaie. L'estampage est la garantie du poids et de la pureté du métal, conformément à l'usage. Et toute modification de la teneur métallique de pièces conservant la même dénomination est une tromperie assimilable à la violation d'une promesse. C'est pourquoi la contrefaçon est «le plus grand crime » et peut être considérée comme une «trahison»: «L'estampage est le reçu public de la valeur intrinsèque ${ }^{60}$. » En politique comme en économie, la confiance collective et le respect des serments sont la clé de voûte d'une organisation réglée et juste de la société et des échanges.

L'image et l'inscription du roi ${ }^{61}$ sur les pièces métalliques sont à comprendre comme la marque du caractère conventionnel de la monnaie. Mais la convention n'est pas arbitraire, et loin de développer un individualisme économique, Locke défend le rôle du consentement collectif contre l'altération et les manipulations monétaires laissées à la discrétion du

58. Quelques considérations, in Locke, 1983a, p. 45; 1991, vol. I, p. 211.

59. Some considerations, in LocKE, 1991, vol. I, p. 283 : "To prey upon the ignorance or necessity of borrowers. »

60. LocKe, 1991, vol. I, p. 307; voir aussi LoCKE, 1991, vol. I, p. 312 : «The counterfeiting the stamp is made the highest crime, and has the weight of treason laid upon it : because the stamp is the publick voucher of the intrinsick value. "

61. LOCKE, 1991, vol. I, p. 328. 
monarque et qui constituent une part importante des politiques économiques du XVII siècle. La même idée prévaut dans sa théorie des taxes et des impôts qu'il faut soustraire à l'arbitraire d'un seul, de même que dans son souci de soumettre la prérogative royale au consentement populaire, ou encore dans sa défense des terres communes en Angleterre ${ }^{62}$. En économie comme en politique, l'alliance de tous doit faire obstacle à la volonté d'un seul, la notion de contrat étant centrale sur les deux terrains et cimentant leur unité : la seule recherche de l'intérêt privé n'est jamais pour Locke, pas plus que pour l'ensemble de la tradition mercantiliste, source d'harmonie sociale. L'échangiste lockien n'est pas l'agent rationnel de la pensée néoclassique, mais inclut la mauvaise part possessive et passionnelle de l'individu oublieux du bien commun.

Concernant ensuite la valeur de la monnaie, Locke s'en prend vigoureusement à tout projet de dévaluation. Certains historiens de la pensée économique lui reprocheront sa naïveté à cet égard et de n'avoir pas compris les possibilités de régulation qui en résultent. Mais le nerf de l'argumentaire de Locke est en l'occurrence de nature éthique et philosophique autant qu'économique: la dévaluation est une tromperie, qui détruit le caractère de garantie publique de l'estampage ${ }^{63}$. On comprend mieux en lisant l'Essai que la politique de maintien de l'étalon répond à la définition de la monnaie comme convention définie par l'usage, et renvoie plus généralement à ce lien social fondateur qu'est le respect des promesses. De même qu'il importe que la signification des mots soit fixée pour que le langage remplisse correctement sa fonction de communication $^{64}$, il faut que ne circulent que des pièces de bon poids, dont l'empreinte corresponde au poids de métal, ou la valeur faciale à la valeur réelle. Le signe authentifie la présence d'une substance, dont la connaissance approfondie n'est pas requise à partir du moment où elle n'est que le support d'une fonction qui, elle, appelle et autorise l'analyse la plus complète possible.

La frappe est donc une opération complexe, qui confère à l'or monnayé le statut de mode mixte, précisément parce que, la teneur en métal étant

62. LocKe, 1994, p. 120, 26 et 104. On peut encore mentionner la décision de Charles II en 1672 de suspendre le remboursement de la dette, décision à laquelle s'opposera vigoureusement Shaftesbury, ainsi que la rumeur qui affirme qu'un souverain catholique confisquera les biens des protestants, voir AsHCRAFT, 1995, p. 32 et 217.

63. Some considerations, in Locke, 1991, vol. I, p. 312. Dans Further considerations, in Locke, 1991, vol. II, p. 429, Locke ironise : "I cannot wonder that Mr. Lowndes, a man so well skill'd in the law, especially of the Mint, the Exchequer and of our money, should all along in this argument speak of clip'd money as if it were the lawful money of England. »

64. Locke, 1983b, p. 410 : «Celui qui n'emploie pas constamment le même signe pour signifier la même idée, mais se sert des mêmes mots tantôt dans un sens, tantôt dans un autre, doit passer dans les écoles et dans les conversations ordinaires pour un homme aussi sincère que celui qui au marché et à la bourse vend différentes choses sous le même nom. » 
garantie, il n'y a plus qu'à se préoccuper de la valeur marchande de celui-ci, en laissant de côté toutes les autres propriétés matérielles de l'or ${ }^{65}$. L'estampage remplace la science de l'or, inutile aux échangistes, pour les assurer néanmoins de la nature substantielle des pièces qu'ils manipulent et pour simplifier les échanges. La convention monétaire, par l'inscription d'un signe à même la matière, vient assurer la liaison de la nature à la convention sociale en articulant un mode mixte sur la substance dont il est l'affection ${ }^{66}$. De ce point de vue, on peut affirmer que la question de l'or et de la monnaie recoupe transversalement l'ensemble de la problématique de l'Essai et, à travers la séquence or métallique-moyen de paiement et réserve de valeur-monnaie fiduciaire, relève successivement de tous les types d'idées que Locke distingue, modes, substances et relations ${ }^{67}$, éclairant l'unité maintenue de ces trois niveaux alors même qu'ils déterminent des domaines de connaissance radicalement distincts. Les idées lockiennes ne sont pas des conventions arbitraires mais des représentations saisies dans leur dépendance à l'égard des choses mêmes, qui en sont les causes. Il importe alors de mesurer de façon exacte leur valeur spécifique de représentation, la monnaie offrant le cas exemplaire d'une dénomination réelle, qui doit impérativement être pensée comme convention pour faire office d'idée adéquate par rapport à la quantité de métal pur qu'elle désigne ${ }^{68}$.

L'opposition économique entre métallisme et cartalisme se dénoue à ce niveau philosophique : toute idée simple est une représentation qu'il faut rattacher non pas à une nature, connue effectivement par là même, mais à une substance matérielle, qui en est la cause nécessaire et inaccessible. La monnaie, réduite par convention aux idées simples d'une quantité et d'une pureté données de métal précieux, ne peut être que vraie:

«Car Dieu ayant, par un effet de sa sagesse, établi de ces idées, comme autant de marques de distinction dans les choses, par où nous puissions être capables

65. Further considerations, in Locke, 1991, vol. II, p. 447 : «Mr. Lowndes say [...] that silver has a price. I answer: silver to silver can have no other price, but quantity for quantity. "

66. LoCKE, 1983b, p. 119: «J'appelle modes ces idées complexes qui, quelque composées qu'elles soient, ne renferment point la supposition de subsister par elles-mêmes, mais sont considérées comme des dépendances ou des affections des substances. » Par ailleurs, défendre la nature strictement conventionnelle de la monnaie aboutit pour Locke à une absurdité, voir Answer to my lords keepers queries, in Locke, 1991, vol. II, p. 396 : «If this could be silver as every one sees might be raised to the value of gold and we might makes ourselves as rich as we pleased."

67. LOCKE, 1983b, p. 118-119.

68. Michael Ayers distingue deux courants de pensée concernant la définition des idées, l'un, aristotélicien et scolastique, l'autre, épicurien et stoïcien, auquel il faut rattacher Locke, voir Ayers, 1993, vol. I, p. 69: «The second, Epicurean line of thought presented a more radical model for intentionality according to which a simple idea is the natural sign or representative of its regular cause. » 
de discerner une chose d'avec une autre, et ainsi de choisir pour notre propre usage, celles dont nous avons besoin ${ }^{69}$. »

L' « espèce » monétaire est fiable, précisément parce qu'elle ne prétend pas être la description d'une substance mais se présente comme la construction manifeste d'une définition unitaire, qui ajoute à la détermination de métal précieux sa fonction d'instrument de circulation et son rôle de réserve de valeur. Élaborée consciemment comme mode mixte, elle devient objet de certitude, à la fois comme moyen pratique d'échange et comme objet théorique. Il semble alors que la monnaie soit le cas paradigmatique de la connaissance modale, l'incarnation des distinctions épistémologiques de l'Essai qui, nous préservant de toute prétention exagérée, autorisent cependant l'organisation savante de la pratique de l'échange et déterminent les conditions et les limites de l'intervention politique. La monnaie est un intermédiaire ontologique autant qu'un instrument économique. Elle renvoie donc à l'articulation générale entre l'organisation sociale et la nature, du moins à ce qu'il est possible et nécessaire d'en savoir, ce qui constitue l'objectif même de l'épistémologie lockienne.

Loin que l'économie se définisse comme une science autonome, elle est pour Locke une connaissance pratique située dans la dépendance directe de la loi de nature, «qui veut la paix et la préservation de tout le genre humain ${ }^{70} \gg$ : via les notions de propriété et de travail, elle renvoie à la définition de l'homme et aux finalités que Dieu a conférées à son œuvre en tant que créateur. Locke affirme très clairement que l'esprit de l'homme est adapté à la recherche du bonheur terrestre, et que cette recherche donne à la technique et à l'économie une importance supérieure à la science de la nature. Il s'agit de :

«[...] trouver de nouvelles inventions ou procédés pour abréger ou faciliter notre labeur; mêler avec sagacité différents agents et matériaux à notre usage, grâce à quoi la quantité de nos richesses (c'est-à-dire des choses utiles aux besoins de notre vie) peut être accrue ou mieux conservée; or l'esprit de l'homme est bien adapté à des découvertes de ce genre, bien que, peut-être, l'essence des choses, leur origine première, les voies cachées de leurs opérations et toute l'étendue des choses corporelles, soient autant au-delà de nos capacités qu'elles nous sont peu utiles. »

Mais Locke précise aussitôt que Dieu a donné une preuve suffisante de son existence par le fait que, lorsque l'homme «possède tout ce que le monde peut lui offrir, il demeure encore insatisfait, inquiet et éloigné du

69. LOCKe, 1983b, p. 310.

70. LOCKE, 1994, p. 7. 
bonheur ${ }^{71} »$. L'effort de construction d'une analyse monétaire rigoureuse est donc l'occasion, non de circonscrire un terrain autonome (celui des échanges marchands), mais bien plutôt de réinsérer un type particulièrement complexe de représentation au sein d'une théorie de la connaissance et d'une philosophie éthique et politique. Aussi spécifiques soient-elles, les catégories économiques employées par Locke ne cessent de renvoyer, au moins implicitement mais souvent expressément, à l'ensemble de sa philosophie, mettant ainsi en évidence l'unité de ses divers secteurs de recherche qui la définissent. Il reste alors à en compléter la démonstration par l'étude du lien essentiel qui existe d'après Locke entre, d'une part, la question de l'échange monétaire et, d'autre part, les questions de la propriété, du travail, et plus généralement de la destination de l'homme.

LA PROPRIÉTÉ, LE TRAVAIL ET LES RICHESSES

Locke est un penseur de l'échange mais nullement l'apôtre de l'accumulation illimitée, même s'il en pense effectivement la possibilité concrète. L'invention monétaire est à cet égard paradoxale : la monnaie, qui permet l'échange comme intermédiaire, s'offre à la thésaurisation en tant qu'elle est réserve de valeur et exacerbe la passion de la propriété. Locke affine l'analyse aristotélicienne en insistant sur le rôle social de la première fonction par opposition au caractère privé de la seconde : l'argent doit être préservé de l'exportation autant que de l'accumulation, qui ont en commun d'entraver la circulation ${ }^{72}$.

Il est évident que la théorie de la monnaie occupe ici encore une situation cruciale : elle assure le lien entre la dimension privée de l'appropriation et de l'échange et le caractère social de l'activité économique pensée comme un tout. Il faut rappeler que l'invention de la monnaie présente un caractère ambivalent pour Locke. L'argent permet d'étendre des possessions qui, sous la forme de marchandises périssables, ne sauraient être accumulées durablement. Mais la corruption des biens matériels est aussi le critère naturel de la quantité qu'il convient de s'approprier : elle proportionne très exactement la quantité des marchandises possédées à « ce qui est nécessaire pour la commodité de la vie ${ }^{73} »$. Après l'apparition de la monnaie, d'une part ce critère naturel ne joue plus, d'autre part l'envie d'accroître toujours davantage ses possessions fait son apparition, tandis que :

71. Journal, 8 février 1677, in Locke, 1990, p. 69 et 70.

72. Some considerations, in LocKe, 1991, vol. I, p. 261.

73. Locke, 1994, p. 27. 
«Lorsqu'il n'existe rien qui soit à la fois durable et rare, et suffisamment précieux pour être accumulé, les hommes ne sont pas disposés à accroître leurs possessions en prenant de nouvelles terres, si riches soient-elles et si libres qu'ils puissent être de les prendre ${ }^{74}$. »

Cette recherche de l'enrichissement donne également lieu à une analyse complexe. Tout d'abord, il faut préciser que Locke ne prend partie ni pour la frugalité extrême, ni pour l'enrichissement sans limite. L'absence de monnaie condamne à une économie de subsistance, tandis que son apparition peut donner naissance à la corruption des mœurs. Le problème n'est donc pas de regretter son émergence mais d'aménager son usage, sachant qu'elle n'inclut pas en elle-même le vrai critère de son utilisation correcte. La monnaie est un moyen qui peut servir plusieurs fins et l'erreur serait précisément de proclamer l'autonomie des phénomènes monétaires en faisant de l'accumulation un but valable par soi-même et de l'échange une régulation sociale quasi mécanique ${ }^{75}$. La solution du problème réside bien évidemment dans la détermination précise de ce qu'il faut entendre par les « commodités de la vie » : au-delà de la satisfaction des besoins vitaux, Locke se prononce pour l'accès général au bien-être et au bonheur. Il faut alors distinguer l'enrichissement privé et l'égoïsme possessif qu'il fait naître de la prospérité nationale et des conditions de sa répartition générale $^{76}$. La difficulté est que l'énergie individuelle consacrée à l'enrichissement est la condition d'une activité économique intense. Là encore, on rencontre une tension de l'analyse, dont Locke assume la présence au travers du concept original de propriété qu'il construit ${ }^{77}$.

Maintenant les réticences — pour ne pas dire la condamnation — antiques et médiévales à l'égard du profit et des richesses, Locke tente d'en

74. LOCKE, 1994, p. 36.

75. On a souvent reproché à Locke, à juste titre d'ailleurs, le caractère statique de son analyse monétaire, voir Hyde Kelly, 1991, p. 92. Locke n'identifie aucun effet autorégulateur propre aux variations du niveau des prix. Au-delà d'une défaillance de l'analyse, on peut affirmer qu'il n'entre pas dans les principes de son analyse d'attribuer aux phénomènes économiques un dynamisme indépendant qui rendrait superflus l'intervention politique et le jugement moral. Toute son analyse se dirige à l'inverse vers la mise en évidence de finalités extérieures et supérieures à la sphère de la circulation, susceptibles d'en orienter et d'en réguler le cours. S'il existe des lois du marché, elles ne s'harmonisent pas selon une boucle de rétroaction mais elles s'ouvrent à chaque instant sur le domaine de l'intervention politique et sociale. C'est pourquoi la question de la définition de la propriété, de sa structure et de sa répartition prend une telle importance dans son œuvre: elle lie les contraintes des lois de l'échange aux exigences de la loi de nature.

76. LOCKE, 1994, p. 98, signale que le pouvoir législatif « ne peut jamais avoir le droit de détruire les sujets, de les réduire en esclavage, ou de les appauvrir à dessein ». Il serait absurde que, de leur côté, les lois du marché soient en mesure de réduire légitimement des hommes à la misère.

77. LoCKE, 1994, p. 90 : les hommes s'unissent «pour la préservation mutuelle de leur vie, de leur liberté et de leurs biens, ce que j'appelle du nom générique de propriété ». 
aménager la rigueur face à la nécessité d'un bien-être collectif croissant. James Tully a mis en évidence la reprise lockienne du concept thomiste de propriété à titre inclusif. Locke maintient, en effet, contre Grotius et Pufendorf, la distinction entre «droit sur » et «droit à », le second se fondant sur le don du monde par Dieu à l'ensemble du genre humain ${ }^{78}$. Par là même, Locke est conduit à défendre l'existence des terres communes contre le mouvement des enclosures, alors même qu'il autorise l'appropriation privée sans limite. Il s'agit pour lui de rendre compatible la propriété collective et la propriété privée des terres au sein d'un même concept juridique. En effet, la multiplication des échanges conduit les hommes à accepter «que la terre soit possédée de manière inégale et disproportionnée ${ }^{79}$ ». Mais cette disproportion peut en venir à menacer l'existence d'une partie du genre humain, incapable d'assurer sa propre conservation. Il convient alors d'ériger la charité en «droit de créance du nécessiteux sur les biens du possédant ${ }^{80} »$. Chaque homme doit pouvoir réaliser par le travail les finalités qui lui sont assignées par Dieu. Les conditions de la vocation sont sociales et économiques, même si l'objectif du salut demeure bien sûr strictement individuel et se situe sur un autre plan, lié au premier mais distinct de lui ${ }^{81}$. Le problème est de permettre à la fois une activité orientée vers l'accroissement du bien-être et de l'appropriation privée, et d'empêcher que cette finalité de rang inférieur, d'une part, ne nuise au niveau individuel à la réalisation de la vocation, d'autre part, n'interdise au plan social la subsistance et le bonheur de tous. Ce système d'exigences individuelles et collectives se répercute tout naturellement au niveau de la monnaie, qui est à la fois issue du consentement commun et l'objet de l'accumulation privée.

À ce niveau, Locke retrouve encore une problématique thomiste, celle du juste prix, mais là aussi modifiée sensiblement par son analyse des règles de l'offre et de la demande. L'article «Venditio » de 1695 examine le cas d'un marchand de blé de Dantzig proposant son blé 5 shillings à Ostende et 20 shillings à Dunkerque où la famine entraîne une forte demande. Est-ce équitable, demande Locke? La justice exige qu'on vende à tous indifféremment au prix du marché. Mais ce prix est défini à l'endroit

78. Tully, 1992, chap. III, IV et v.

79. LOCKE, 1994, p. 37.

80. Tully, 1992, p. 188.

81. DunN, 1991, p. 60, parle à cet égard de l'individualisme religieux de Locke. Il ne saurait constituer le fondement de l'individualisme libéral que lui attribuent Louis Dumont ou Crawford B. Macpherson. Concernant les origines religieuses de la question, SKINNER, 1978, p. 328, note : «Like Locke a century later, the Huguenots assume that amongst the things we may be said to have the freedom and thus the right to dispose of within the bounds of the laws of nature are those properties - as we still punningly call them - which are intrinsic to our personalities, and in particular our lives and liberties. » 
même où l'on vend. À la recommandation thomiste de modération mutuelle ${ }^{82}$, Locke substitue l'affirmation que «la mesure commune au vendeur et à l'acheteur est simplement que si l'un vend aussi cher qu'il peut au marché, l'autre achète aussi bon marché qu'il peut ${ }^{83}{ }$. Le rapport entre l'offre et la demande définit automatiquement la valeur de la marchandise sans supprimer pour autant l'impératif ultime de charité à l'égard de ceux qui se trouvent dans la nécessité la plus pressante : et Locke prend soin d'ajouter que celui «qui emporte son blé parce qu'ils ne veulent pas lui donner plus qu'ils n'en sont capables [...] est sans aucun doute coupable de meurtre ${ }^{84} \gg$. L'impératif de charité vient cette fois s'adjoindre du dehors et comme cas limite à la fixation des prix par le marché. Il n'en reste pas moins que la circulation générale des marchandises demeure ainsi placée sous la juridiction ultime de la loi de nature ${ }^{85}$.

L'offre et la demande aident à déterminer le prix des biens échangés mais ils ne sont pas la source de la valeur, qui réside en amont du marché, dans la nature et le travail. Cette thèse de la double origine de la richesse est un emprunt à la Genèse, devenu un leitmotiv de la pensée économique ${ }^{86}$. Mais Locke instaure une franche inégalité entre les deux éléments, puisqu'il va jusqu'à affirmer que dans les dépenses nécessaires à la production des choses, «les quatre-vingt-dix-neuf centièmes doivent être attribués au travail ${ }^{87}$ ». L'estimation vise à démontrer que «bien que les choses de la nature soient données en commun, l'homme [a] cependant parce qu'il est maître de lui-même et propriétaire de sa propre personne et des actions ou du travail de cette même personne - en lui-même le grand fondement de la propriété ${ }^{88} \gg$. La propriété et le travail se conditionnent mutuellement mais renvoient ultimement au salut et à la nécessité continue

82. Thomas D'Aquin, 1926, t. III, IIa-IIae, q. 77, p. 485 : « Si l'acheteur tire un grand avantage de ce qu'il reçoit du vendeur, et que ce dernier ne subisse aucun préjudice en s'en défaisant, il ne doit pas vendre au-dessus de sa valeur. »

83. "Venditio », in Locke, 1991, vol. II, p. 499: «The measure that is common to buyer and seller is just that if one should buy as cheap as he could in the market the other should sell as dear as he could there. "

84. LocKe, 1991, vol. II, p. 499 : "If he carry it away unlesse the will give him more than they are able [...], he is no doubt guilty of murder. » Par ailleurs, dans For a generall naturalization, in LocKe, 1991, vol. II, p. 492, Locke est parfaitement hostile à l'aide systématique apportée aux pauvres et souhaite qu'ils «n'aient ni la permission, ni l'encouragement d'être oisifs ».

85. Locke, 1992a, p. 145, conseille de former les enfants à une bonne compréhension de la propriété en leur enseignant «la libéralité, l'empressement de partager avec les autres ce qu'ils possèdent ou ce qu'ils aiment ».

86. Vidonne, 1986, p. 14-15, signale qu'on la trouve chez Hobbes, Cantillon, Boisguilbert, Quesnay, Diderot, Bentham, Storch, Malthus et Marx.

87. LOCKE, 1994, p. 31.

88. LOCKE, 1994, p. 34. 
d'en produire les signes ${ }^{89}$, qui se double de l'objectif terrestre de la prospérité collective : «Toutes nos affaires se traitent ici-bas ${ }^{90}$. »

Cette conception éthique et théologique du travail donne naissance à un curieux texte, qui interdit qu'on voie en Locke un précurseur de l'organisation capitaliste du travail. L'article « Labor» de 1693 débute par l'affirmation suivante : «Nous devons considérer comme une marque de la bonté de Dieu qu'il nous ait placés en cette vie sous la nécessité du travail ${ }^{91}{ }^{\text {}}$ et propose, bien avant Paul Lafargue, une répartition plus égalitaire du travail manuel et du travail intellectuel, compte cependant tenu « de la distinction qui doit exister entre les rangs ${ }^{92} \gg$. La proposition initiale de 6 heures quotidiennes de travail manuel pour tous se transforme alors en 9 heures de travail et 3 heures d'étude pour les travailleurs manuels, et en la proportion inverse pour les gentlemen et les savants. Si cette correction est plus qu'une concession à une hiérarchie sociale que Locke ne souhaite nullement bouleverser, le but du travail apparaît clairement et il est évident qu'il ne vise qu'à titre annexe l'enrichissement : «Si le travail dans le monde était correctement organisé et réparti, il y aurait plus de science, de paix, de santé et d'abondance qu'aujourd'hui. Et l'humanité serait beaucoup plus heureuse qu'à présent ${ }^{93}$. » Il n'est pas étonnant que Locke ne se préoccupe nullement de la distinction entre travail productif et travail improductif élaborée à la même époque par William Petty : son but est à la fois éthique, religieux et économique. Locke se définit volontiers lui-même comme un ouvrier et on peut être frappé par les occurrences de la métaphore qui rapproche la recherche du savoir de la découverte d'un filon d'or ${ }^{94}$ et de sa prospection. La récompense terrestre est un signe et une gratification secondaire pour un effort dont la portée outrepasse largement le bénéfice immédiat.

Les questions économiques sont bien inséparables des finalités éthiques et religieuses de l'homme. Qu'elles possèdent leur spécificité n'annule en rien leur subordination en dernière instance à l'égard de fins d'une autre nature qu'elles. Cet aspect se montre encore dans l'analyse de la richesse et de la puissance des nations que propose Locke. Ici encore, l'analyse subit la tension forte de ses multiples motifs et enjeux : la prospérité anglaise est un but restreint qui est, en partie au moins, incompatible avec le développement des autres nations et donc de l'humanité dans son ensemble. Locke ne

89. DunN, 1991, p. 223-225.

90. Journal, in LocKe, 1990, p. 70.

91. "Labor », in Locke, 1991, vol. II, p. 493 : «We ought to looke on it as a marke of goodnesse in god that he has put us in this life under a necessity of labour.»

92. LocKe, 1991, vol. II, p. 494 : «The distinction that ought to be in ranks of men.»

93. Locke, 1991, vol. II, p. 495 : «If the labour of the world were rightly directed and distributed there would be more knowledge peace health and plenty in it than now there is. And man kinde be much more happy than now it is. »

94. LocKe, 1986, p. $37 ; 1983$ b, p. $57 ; 1975$, p. 21. 
peut qu'être pris en tenaille entre l'universalisme chrétien et l'exclusivisme national propre au mercantilisme. Dans le cadre de la rivalité de l'Angleterre avec les Provinces-Unies en particulier et des guerres qui les opposent, le débat économique porte sur les moyens de faire efficacement concurrence aux marchands hollandais ${ }^{95}$. La circulation mondiale des richesses est pensée comme un jeu à somme nulle, où les gains des uns sont obligatoirement les pertes des autres :

«Dans un pays non pourvu en mines, il n'est que deux manières de devenir riche, les conquêtes ou le commerce [...] Nul n'est assez vain pour entretenir l'illusion que nous pouvons moissonner au fil de nos épées les profits du monde [...] Le commerce reste donc la seule façon que nous ayons d'accéder aux richesses ${ }^{96}$.»

L'Angleterre connaît une importante crise de la circulation due au manque de liquidités et c'est dans ce contexte que le ministère whig sera conduit à solliciter en 1695 l'avis et les propositions de Locke.

Celui-ci reprend l'idée admise à l'époque que le commerce à la fois fonctionne selon des lois propres ${ }^{97}$ et qu'il est un moyen de la puissance politique. Au total, la prospérité d'une nation s'estime donc en fonction de sa situation dans la circulation mondiale de la monnaie et des biens : il lui faut capter la plus grande part possible du stock existant de métaux précieux. Les questions monétaires se situent donc aussi sur le plan de la politique internationale : la monnaie pour Locke dynamise le commerce en stimulant la demande, et de ce point de vue il se range aux côtés de Thomas Mun, pour qui il est impossible de séparer phénomènes réels et phénomènes monétaires ${ }^{98}$. La prospérité nationale suppose une politique monétaire rigoureuse ainsi qu'une planification étatique pour que le commerce du marchand profite à la société dans son ensemble ${ }^{99}$. L'économie lockienne est bien, avant tout, une politique de l'économie.

95. Shaftesbury prononce en 1673 devant le Parlement un discours demeuré célèbre, «Delenda est Carthago », pour obtenir son soutien dans la troisième guerre contre les PaysBas. Il définit alors cette guerre comme « une compétition [...] pour le commerce du monde entier », voir AsHCRAFT, 1995, p. 123.

96. Quelques considérations, in Locke, 1983a, p. 57-58 ; Some considerations, in Locke, 1991, vol. I, p. 222.

97. Some considerations, in Locke, 1991, vol. I, p. 282 : «The price of things will not be regulated by laws, though the endeavours after it will be sure to prejudice and inconvenience trade, and put your affairs out of orders. » (Le prix des choses ne peut être réglé par les lois et les efforts pour le faire cependant porteront à coup sûr préjudice et tort au commerce, et désorganiseront vos affaires.)

98. Steiner, 1992, $2^{\mathrm{e}}$ part.

99. LAZZERI, 1992, p. 95 : «Rien ne garantit que le droit à la conservation collective sera d'autant mieux assuré qu'il dépendra de l'initiative et des aptitudes des possédants. De ce point de vue, il n'est pas certain que la difficulté à laquelle se trouve confronté Locke ait été résolue - malgré son raffinement théorique — par la théorie politique et l'économie libérale ultérieures. » 
Le mercantilisme, par-delà la diversité des auteurs qu'on range sous cette bannière, n'oppose pas l'intervention étatique et le libre-échange, mais entend par liberté du commerce l'exercice d'une protection judicieuse de l'État sous la forme d'un système de privilèges dont le modèle demeure le Navigation Act de $1651^{100}$. Le thème de la balance du commerce reflète cette volonté de déceler les lois propres du négoce tout en faisant l'objet d'une politique économique d'autant plus efficace qu'elle sera au fait des lois de l'échange. Chez Locke, ce souci s'exprime au travers de sa conception de l'intérêt et du monnayage, jamais séparée de ses autres préoccupations : une politique monétaire efficace stimule l'activité et permet la mise au travail de l'ensemble de la population, ainsi, par suite, que l'essor démographique, condition de la vraie richesse ${ }^{101}$. L'augmentation de l'activité n'est pas synonyme d'accroissement de la productivité et le problème de la mise au travail des pauvres et de la discipline qu'il faut parvenir à leur imposer ne se situe qu'aux marges de ce qui préoccupera l'économie politique classique : elle ne donne pas lieu à une réflexion sur l'organisation sociale de la production mais associe immédiatement et mécaniquement prospérité nationale et essor démographique. On retrouve à ce niveau la thèse mercantiliste des avantages financiers et militaires que procure une population nationale relativement plus élevée que dans les autres pays, et qui conduit Locke à défendre des mesures d'incitation à l'immigration, notamment française, en Angleterre ${ }^{102}$.

On doit constater que Locke est à la fois interventionniste et prudent quant aux conditions et aux limites des décisions politiques sur le terrain des échanges commerciaux. Il existe une causalité économique qui définit la place et la nature des actions dans ce domaine, et c'est en ce point que théorie monétaire et fixation du taux d'intérêt se rencontrent. Locke est hostile à une baisse autoritaire du taux d'intérêt, censée favoriser l'investissement. La raison en est que l'intérêt est un prix, celui du loyer de l'argent, et qu'il se détermine comme pour toutes les marchandises par le rapport entre l'offre et la demande : le bas taux de l'intérêt hollandais, pris comme modèle par Child, est selon Locke un résultat et non une cause de la prospérité. Et il élabore à cette occasion sa théorie de la double valeur de la monnaie, en distinguant le prix payé pour l'emprunter de la valeur de la monnaie échangée contre des biens. Ne parvenant pas à relier ces deux dimensions, il se résout à une théorie économique délibérément lacunaire,

100. Steiner, 1992, p. 134 : les seuls défenseurs du libre-échangisme se recruteront alors du côté des marchands interlopers, opposés aux grandes compagnies dont les privilèges leur portent préjudice et qui souhaitent une concurrence accrue.

101. LOCKE, 1994, p. 32 : «Il convient de préférer l'abondance de la population à l'étendue des possessions, et [...] l'augmentation des terres cultivées et le bon emploi que l'on en fait constituent le grand art du gouvernement. »

102. For a generall naturalization, in Locke, 1991, vol. II, p. 489. 
en même temps qu'il laisse alors toute sa place à la dimension politique et sociale de la question : il est frappant de voir comment son analyse s'effectue du point de vue des diverses classes sociales en présence, Locke restant au total plus proche des propriétaires fonciers que des banquiers et des marchands, qui «sont les derniers à être affamés ${ }^{103}$ ». C'est à ce niveau social que les choix économiques trouvent leurs motifs déterminants: Locke signale qu'une dévaluation porte préjudice en premier lieu à tous les créanciers ainsi qu'aux propriétaires fonciers. Par ailleurs, l'analyse conserve toujours sa dimension éthique : Locke n'hésite pas à attribuer la baisse du prix de la terre aux dettes dont sont couverts des propriétaires s'abandonnant à la débauche et ayant renoncé à « la sobriété, la frugalité et l'industrie » du règne d'Elisabeth ${ }^{104}$. La transition est d'autant plus aisée de la sphère économique au domaine moral et religieux que Locke maintient l'assimilation aristotélicienne de l'économie nationale à l'économie domestique : «Un royaume devient riche ou pauvre, comme un fermier le devient et pas autrement ${ }^{105}$. » L'équilibre budgétaire est la condition de la survie, et l'excédent de la balance commerciale celle de la prospérité.

Par suite, le commerce international est privilégié au détriment de la consommation intérieure, appauvrissante. La plupart des théoriciens de cette période réclament également des bas salaires, qui ont le mérite d'interdire l'oisiveté, de limiter les dépenses des travailleurs et d'abaisser les prix des marchandises. Dans la situation anglaise de sous-emploi ${ }^{106}$, l'urgence est de contraindre les pauvres au travail alors même que l'intérêt économique ne motive pas ou peu leur comportement. Si Locke accorde au peuple des droits politiques, conformément aux conceptions de l'aile la plus radicale du mouvement whig, il fait partie de ceux qui dénoncent l'oisiveté des pauvres et préconisent l'organisation des workhouses en même temps que le maintien de bas salaires ${ }^{107}$.

Il en vient alors à distinguer deux sous-catégories dans le peuple : d'une part, ceux qui contribuent au commerce, et tout particulièrement au commerce extérieur; d'autre part, les paresseux, parmi lesquels il compte indistinctement les domestiques, les mendiants, les détaillants, les hommes de loi et les soldats ${ }^{108}$. La séparation des deux classes s'effectue du point de vue de l'utilité commune en même temps qu'en fonction de la capacité des hommes à assurer leur subsistance par leur propre travail. Locke peut ainsi

103. Some considerations, in LockE, 1991, vol. I, p. 271 : «The merchant, and monied man [...] will be sure to starve last.»

104. Locke, 1991, vol. I, p. 269 : «Sobriety, frugality, and industry.»

105. LOCKE, 1983a, p. 66; 1991, vol. I, p. 230.

106. Hill et Postan, 1977, p. 314.

107. "Trade », in LOCKE, 1991, vol. II, p. 486.

108. LOCKE, 1991, vol. II, p. 485 : «Reteiners of gentry and beggers or which is worth hinder trade as retailers in some degree. Multitudes of lawyers, but above all soldiers in pay.» 
à la fois défendre la vertu de charité et regretter les « lois très inopportunes ${ }^{109} \gg$ de protection des pauvres, qui seront bientôt au centre du débat économique en Angleterre. Le magistrat peut même être conduit à interdire l'exercice de la charité quand elle incite à la paresse ${ }^{110}$, mais on aurait tort d'y reconnaître un motif libéral : les vertus individuelles sont à estimer aussi par rapport au bien commun, dans la mesure où elles modifient la réalité sociale et retentissent sur les comportements privés. À l'avantage économique d'un faible coût de production, le bas prix du travail ajoute donc le bénéfice éthique de l'obligation au labeur et à la frugalité, vertus dont Locke regrette la disparition chez les classes possédantes. L'appel à l'essor du commerce est au centre d'une axiologie d'inspiration puritaine dont il demeure inséparable, même si le système des valeurs éthiques se trouve manifestement de son côté adapté à la perspective d'un niveau d'activité toujours croissant.

Le commerce est un problème essentiel pour Locke, mais s'il ne le coupe pas de ses tenants et aboutissants politiques, il ne sépare pas davantage la circulation de la production des biens et de la perspective générale d'un accroissement continu de l'activité. Cette production est avant tout agricole et les questions liées à la terre sont en effet au centre des préoccupations de Locke, qui voit dans le développement de l'agriculture la condition fondamentale de la prospérité publique ${ }^{111}$ : la chute des rentes est à déplorer. Locke précise qu'elles baisseront jusqu'à ce que « l'industrie et la frugalité, jointes à un commerce bien organisé, rétablissent la richesse et l'opulence que le royaume connaissait autrefois ${ }^{112}{ }^{»}$. Locke n'anticipe pas sur le développement de l'industrie et des manufactures, à telle enseigne qu'il s'efforce de penser l'usure et le profit sur le modèle de la rente pour en légitimer l'existence, cette fois à l'encontre de leur condamnation médiévale ${ }^{113}$. S'il justifie le prêt à intérêt, il reste bien loin de l'idée de capital et de profit : la monnaie doit toujours être remise en circulation pour jouer son rôle de stimulateur du commerce, mais elle ne saurait être accumulée sous la forme d'un capital circulant qui possède son autonomie et sa fonction spécifique. Elle demeure avant tout un moyen d'échange et la défense lockienne des propriétaires fonciers par rapport aux marchands et aux banquiers n'est que l'expression sociale de cette stricte hiérarchisation des fonctions économiques.

109. For a generall naturalization, in Locke, 1991, vol. II, p. 489-490 : «Very inconvenient laws for mainteining the poor.»

110. Essai sur la tolérance, in Locke, 1992b, p. 118.

111. L'Angleterre est un pays où « la terre est le plus grand fond », voir Some considerations, in LocKe, 1991, vol. I, p. 272 : «Their great fund is in land.»

112. Some considerations, in Locke, 1991, vol. I, p. 272 : «Till general industry, and frugality join'd to a well order'd trade, shall restore to the Kingdom the riches and wealth he had formerly. "

113. MARX, 1977b, t. III, p. 571. 
La sphère de la circulation est bien un intermédiaire et un moyen dans la recherche de la prospérité : l'insistance de Locke et de tous les théoriciens de cette époque sur l'importance des phénomènes monétaires et de la sphère de la circulation ne doit pas faire illusion. On assiste moins à l'émergence d'un concept embryonnaire de capital qu'à l'identification du niveau qui donne le plus de prise à l'intervention étatique. On peut, bien sûr, trouver moderne l'idée que la monnaie est une médiation qui détermine ou conditionne les sphères qu'elle relie, mais il ne faut pas inverser l'ordre des priorités. Locke le dit très explicitement : «Le négoce est donc nécessaire à la production des richesses, et l'argent à la poursuite du négoce », ou encore :

«L'excédent du négoce entre nous et nos voisins doit inévitablement emporter notre argent et rapidement nous laisser pauvres et vulnérables. L'or et l'argent, quoiqu'ils soient utiles à peu de monde néanmoins commandent toutes les commodités de la vie; et donc c'est dans leur abondance que consistent les richesses ${ }^{114}$.»

On ne saurait mieux dire que l'argent occupe une fonction décisive pour l'enrichissement, mais qu'il n'est la richesse qu'à la condition de circuler et de faire circuler les marchandises.

Locke rend sans doute possible la prochaine réorientation de la pensée économique en direction de la production et le passage de l'analyse de l'intérêt à celle du profit. Mais la question de l'échange doit être avant tout pensée en corrélation avec la définition du travail comme devoir et la mention des «nécessités et commodités de la vie » comme constituant ses finalités individuelles et collectives. S'il justifie bien l'appropriation privée, il maintient en même temps la catégorie de propriété indivise. En ce sens, il paraît problématique d'affirmer qu'il prépare le terrain au libéralisme : rien dans son analyse ne décrit une réalité qui ne s'installera de façon identifiable que bien plus tard. Par contre, Locke est sensible aux différents intérêts en présence et à leurs conflits plus ou moins larvés dans une Angleterre en proie aux bouleversements politiques les plus profonds ${ }^{115}$. On a le sentiment, à lire les textes économiques de Locke, qu'il pense surtout l'économie comme le terrain d'affrontement de plusieurs conceptions et qu'elle constitue un lieu privilégié, à la fois théorique et pratique, de transformation sociale et politique.

114. Locke, 1983a, p. 58 et 56; 1991, vol. I, p. 223-224 et 221.

115. Some considerations, in LockE, 1991, vol. I, p. 290-291 : Locke examine les effets politiques de la chute des rentes sur les différentes «classes » sociales, en indiquant par exemple que les laboureurs réduits à la misère seront amenés à prendre les armes contre les riches, tandis que les landed men entreront en lutte à la fois contre les marchands et les banquiers. 
Si son analyse économique exprime directement et de façon complexe ses partis pris éthiques, religieux et politiques, elle présente une certaine indétermination qu'on a souvent mise au compte de l'incohérence de la pensée lockienne. Il semble plutôt que Locke entrevoie la possibilité de plusieurs perspectives historiques, pas nécessairement compatibles entre elles d'ailleurs, mais qui sont autant de voies non libérales de développement. Que ces voies n'eussent guère été praticables n'est pas la question; qu'on les évalue à la lumière de la direction effectivement prise par l'économie anglaise et européenne est un contresens, et concernant les intentions de Locke, et concernant ce qui détermine effectivement telle ou telle transformation économique de grande ampleur. Le défaut majeur d'une telle lecture est de masquer la spécificité de l'approche lockienne des phénomènes économiques et monétaires derrière la conviction que l'économie serait une science qui tâtonne d'abord à la recherche de son autonomie et ne fait, dans un premier temps, qu'esquisser ou pressentir les concepts centraux de l'économie politique classique et contemporaine. C'est ainsi que, piétinant au seuil de la maturité scientifique, Locke aurait entrevu la notion de «travail accumulé », ou encore la fonction de régulation des taux d'intérêt, ou bien la " préférence pour la liquidité », voire même l' « exploitation du travail ${ }^{116}$. Décrire Locke comme un protolibéral encore marqué des vestiges " préscientifiques ${ }^{117}$ » du mercantilisme, c'est oublier qu'il n'a jamais envisagé qu'il puisse exister une science autonome nommée économie et accompagnant le développement d'un nouveau mode de production, le capitalisme ${ }^{118}$. Une telle science manifeste elle-même un choix théorique et pratique, qui ne résulte pas seulement des circonstances historiques objectives qui lui donnent naissance mais aussi d'une conception politique et philosophique précise, que masque parfois, justement, l'affirmation d'une autonomie advenue de la science économique.

Il reste à se demander comment Locke a pu être l'objet d'une lecture si partiale. Concernant la seule histoire de la pensée économique, que Locke soit un tenant de la théorie quantitative semble avoir été la cause de son rattachement ultérieur à la tradition classique et néoclassique, mention étant faite alors de ses incohérences et de ses contradictions. Pourtant, la théorie

116. Respectivement : Hyde Kelly, 1991, p. 75 ; Guggenheim, 1978, p. 35 ; Keynes, 1979 , p. 337-339; MARX, 1974, p. 430-431. Keynes, 1979, p. 339, qui rend au mercantilisme sa dignité théorique, décrit Locke comme ayant « un pied dans le monde mercantiliste et l'autre dans le monde classique ». Le danger est alors de lire son œuvre comme une transition sans véritable consistance propre, comme un passage défini seulement et rétrospectivement par ce à quoi il conduit.

117. SCHUMPETER, 1983, p. 485.

118. Malgré sa prudence, la lecture de Wood, 1984, p. 114, qui voit en Locke un théoricien du capitalisme agraire en formation de cette époque et de lui seul, retrouve pourtant la tradition d'interprétation rétrospective, en affirmant in fine que Locke est « in a broader sense, $a$ pioneer of the spirit of capitalism $»$. 
quantitative est moins un fil continu d'une pensée économique à la recherche de son autonomie et en voie de mathématisation, qu'une thèse qui n'implique pas forcément une théorie de l'équilibre général ou l'affirmation de la neutralité de la monnaie. Locke s'efforce de penser la monnaie dans sa spécificité et son rôle propre, en faisant non pas de la richesse privée mais de la richesse sociale l'objet de l'économie politique ${ }^{119}$. En ce sens, la monnaie n'est nullement une réalité séparée, mais un foyer théorique où se réfractent les multiples dimensions de l'analyse, politique, juridique, éthique, philosophique et religieuse : on s'est efforcé de le montrer, l'or chez Locke renvoie tout à la fois au langage et à la connaissance, à l'instrument monétaire, au travail, à la nature créée par Dieu, à la répartition des richesses, à l'éducation, à l'autorité publique, au consentement collectif, au contrat et au respect des promesses, aux relations internationales.

La monnaie, peu à peu exclue de l'analyse économique ultérieure, n'est pas pour Locke un objet spécial, ou le voile neutre qu'y verra Jean-Baptiste Say, mais un ensemble de règles sociales qui interdisent que l'économie prétende s'émanciper de la politique. Il semblerait donc que ce soit la capacité d'une théorie à intégrer les dimensions multiples qu'on a mentionnées, en les faisant converger en un point unique, la monnaie, qui constitue l'économie politique comme telle, même si cette dernière n'a cessé de réécrire son histoire sous la forme d'une odyssée de la valeur. C'est donc, très logiquement, au moment de la naissance de cette pensée économique que sa complexité intrinsèque s'aperçoit le mieux et que ses enjeux s'exposent le plus clairement. Ultime paradoxe, la véritable modernité de Locke résiderait alors précisément dans le fait qu'il n'est pas un précurseur du libéralisme économique mais bien le partisan d'une approche politique de l'économie, qui contribue à éclairer sous l'angle de ses choix fondamentaux, devenus plus difficilement discernables, la théorisation ultérieure.

Isabelle GARO (juillet 1998).

119. Concernant l'ensemble du mercantilisme, c'est la thèse que défend CARTelier, 1996, p. 66 . 


\section{LISTE DES RÉFÉRENCES}

AshCRAft (Richard), 1995, La Politique révolutionnaire et les deux traités du gouvernement de John Locke, trad. Jean-François Baillon, Paris, Presses universitaires de France.

AYERs (Michael), 1993, Locke, epistemology and ontology, Londres, Routledge, 2 vol.

Blaug (Mark), 1987, La Pensée économique. Origine et développement, $4^{\mathrm{e}}$ éd., trad. Alain et Christiane Alcouffe, Paris, Economica.

Braudel (Fernand), 1979, Civilisation matérielle, économie et capitalisme, $x v^{e}$ XVIII $I^{e}$ siècle, Paris, Armand Colin.

CAFFentzis (Constantine George), 1989, Clipped coins abused words and civil government. John Locke's philosophy of money, New York, Autonomedia.

Cartelier (Jean), 1996, La Monnaie, Paris, Flammarion.

DANG (Ai-Thu), 1997, "Juste prix et science de l'enrichissement chez John Locke », Economies et sociétés, Economia, Histoire de la pensée économique, sér. P.E. $\mathrm{n}^{\circ} 25$ (Cahiers de l'ISMEA-PUG), 1, 1997, p. 49-69.

Dumont (Louis), 1977, Homo Aequalis. Vol. I : Genèse et épanouissement de l'idéologie économique, Paris, Gallimard.

Dunn (John), 1991, La Pensée politique de John Locke, trad. Jean-François BAILLON, Paris, Presses universitaires de France.

Guggenheim (Thomas), 1978, Les Théories monétaires préclassiques, Genève, Droz.

Hill (Christopher) et Postan (Michael), 1977, Histoire économique et sociale de la Grande-Bretagne. T. I : Des origines au XVIII siècle, trad. Claude BERTRAND, Paris, Seuil.

Hyde Kelly (Patrick), 1991, « General introduction », in Locke on money, Oxford, Clarendon Press, vol. I, p. 1-109.

Keynes (John Maynard), 1971, Théorie générale de l'emploi, de l'intérêt et de la monnaie, trad. Jean de LaRgentaye, Paris, Payot.

LAzzeri (Christian), 1992, «Le droit de résistance dans la philosophie libérale. Le cas de Locke », Rue Descartes, 3, p. 79-95.

Locke (John), 1975, De la conduite de l'entendement, $1^{\mathrm{re}}$ éd. 1671, ici trad. Yves Michaud, Paris, Vrin.

Locke (J.), 1983a, Pièces diverses de monsieur John Locke, $1^{\mathrm{re}}$ éd. 1691, ici trad. Paulette TAÏEB, Paris, MAUSS.

Locke (J.), 1983b, Essai philosophique concernant l'entendement humain, $1^{\mathrm{re}}$ éd. 1706, ici trad. Pierre Coste, Paris, Vrin.

LocKe (J.), 1984, Le Magistrat civil, $1^{\text {re }}$ éd. 1660, ici trad. René FréREux, centre de philosophie politique et juridique, université de Caen.

LOCKE (J.), 1986, Essais sur la loi de nature, $1^{\mathrm{re}}$ éd. 1664, ici trad. Hervé GuINERET, bibliothèque de philosophie politique et juridique, université de Caen.

Locke (J.), 1990, Morale et loi naturelle, $1^{\text {re }}$ éd. 1676-1693, ici trad. Jean-Fabien SPITz, Paris, Vrin. 


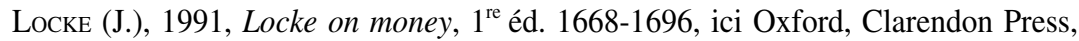
2 vol.

Locke (J.), 1992a, Quelques pensées sur l'éducation, $1^{\mathrm{re}}$ éd. 1693, ici trad. Gabriel Compayré, Paris, Vrin.

Locke (J.), 1992b, Lettre sur la tolérance, $1^{\text {re }}$ éd. 1667-1686, ici trad. Jean Le CleRC et Jean-Fabien Spitz, Paris, Garnier Flammarion.

Locke (J.), 1994, Second traité du gouvernement, $1^{\text {re }}$ éd. 1690, ici trad. Jean-Fabien SPITZ, Paris, Presses universitaires de France.

MACPHERSON (Crawford B.), 1973, La Théorie politique et l'individualisme possessif de Hobbes à Locke, trad. Michel Fuchs, Paris, Gallimard.

MARX (Karl), 1974, Théories sur la plus-value, $1^{\text {re }}$ éd. 1863, t. I, ici trad. dir. par Gilbert BADIA, Paris, Éditions sociales.

Marx (K.), 1977a, Contribution à la critique de l'économie politique, $1^{\mathrm{re}}$ éd. 1859, ici trad. Maurice Husson et Gilbert BADIA, Paris, Éditions sociales.

Marx (K.), 1977b, Le Capital, $1^{\text {re }}$ éd. 1894, t. III, ici trad. Gilbert BADIA, Paris, Éditions sociales.

Pocock (John), 1980, «The myth of John Locke and the obsession with liberalism », in John Locke, sous la dir. de John Pocock et Richard AsHCRAFT, Berkeley, University of California Press.

Poсоск (J.), 1997, Le Moment machiavélien, trad. Luc Bonot, Paris, Presses universitaires de France.

Pribram (Karl), 1986, Les Fondements de la pensée économique, trad. H. P. BerTRAND, Paris, Economica.

Ruffini (Pierre-Bruno), 1996, Les Théories monétaires, Paris, Seuil.

Schumpeter (Joseph), 1983, Histoire de l'analyse économique. T. I : L'âge des fondateurs, Paris, Gallimard.

SKInNER (Quentin), 1978, The Foundations of modern political thought. T. I : The age of Reformation, Cambridge, Cambridge University Press.

SPITz (Jean-Fabien), 1994a, Introduction au Second traité du gouvernement, Paris, Presses universitaires de France.

SpITZ (J.-F.), 1994b, « John Locke, père fondateur du libéralisme? », in Les Paradigmes de la démocratie, dir. Jacques BIDET, Paris, Presses universitaires de France (Actuel Marx-Confrontation), p. 227-250.

STEINER (Philippe), 1992, "Marchands et princes. Les auteurs dits "mercantilistes" », in Nouvelle histoire de la pensée économique. T. I : Des scolastiques aux classiques, sous la dir. de Alain Béraud et Gilbert Faccarello, Paris, La Découverte, p. 95-140.

Strauss (Léo), 1986, Droit naturel et histoire, trad. Monique Nathan et Éric DE DAMPIERRE, Paris, Flammarion.

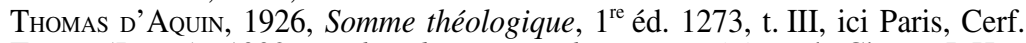

Tully (James), 1992, Locke, droit naturel et propriété, trad. Chaïm J. Hutner, Paris, Presses universitaires de France.

Vidonne (Paul), 1986, La Formation de la pensée économique, Paris, Economica. VILAR (Pierre), 1974, Or et monnaie dans l'histoire, Paris, Flammarion.

Wood (Neal), 1984, John Locke and agrarian capitalism, Berkeley, University of California Press. 